\title{
Ability of Glasgow Coma Scale and Full Outline of Unresponsiveness Score in Measuring Level of Consciousness and Outcome in Patients Receiving Sedation Under Mechanical Ventilation
}

\author{
Aliakbar Keykha, ${ }^{1,}$ Hasan Askari, ${ }^{1}$ Ali Navidian, ${ }^{1}$ and Bibi Mahdie Khodadadi Hosseini ${ }^{2}$ \\ ${ }^{1}$ Community Nursing Research Center, Zahedan University of Medical Sciences, Zahedan, IR Iran \\ ${ }^{2}$ Bachelor of Nursing, Alzahra Eye Hospital, Zahedan University of Medical Sciences, Zahedan, IR Iran \\ "Corresponding author: Aliakbar Keykha, Community Nursing Research Center, Zahedan University of Medical Sciences, Zahedan, IR Iran. E-mail: aliakbar.keykha@gmail.com
}

Received 2016 December 24; Revised 2017 February 01; Accepted 2017 February 19.

\begin{abstract}
Background: Determining the level of consciousness is one of the main responsibilities of nurses in intensive care unit (ICUs). Scales used to determine the level of consciousness should be less affected by the injection of analgesics and sedatives and should provide proper vision of the patient's status.

Objectives: This study was conducted to assess the ability of two tools including Glasgow coma scale (GCS) and full outline of unresponsiveness (FOUR) score in measuring the level of consciousness and outcome in patients receiving sedation under mechanical ventilation.

Methods: The present analytical descriptive study was conducted on 80 trauma patients receiving sedation under mechanical ventilation in 2016 in Zahedan, Iran. The patients having inclusion criteria were evaluated using the two tolls of GCS and FOUR score on admission and after starting and stopping sedation until the last days of hospitalization. The data were analyzed to assess the ability of the tools in measuring the level of consciousness after starting sedation and determine the outcome using independent t-test, chi-square test, and repeated measures ANOVA.

Results: Of 80 patients studied, 61 were male (76.2 \%) and the rest were female. Independent t-test results showed no significant association between the mean admission GCS score and mortality though the deceased patients obtained higher mean score $(\mathrm{P}=$ 0.978). Independent t-test revealed no significant association between the mean FOUR score on the first-day and mortality, though the deceased patients gained higher mean score $(P=0.578)$. The results of repeated measures ANOVA indicated that the mean GCS score after onset of sedative infusions reduced significantly compared to earlier $(\mathrm{P}=0.001)$. This test also revealed that the mean FOUR score after onset of sedation decreased slightly than before although this reduction was not statistically significant $(\mathrm{P}=0.432)$. Conclusions: According to fewer changes in FOUR score than the GCS score after starting and stopping sedation, it is more appropriate criterion for measuring the level of consciousness in trauma patients receiving sedation.
\end{abstract}

Keywords: Glasgow Coma Scale, FOUR Score, Sedative Infusions, Trauma Patients

\section{Background}

The intensive care unit (ICU) is a unit at hospital where seriously ill patients are cared because of serious lung disease, brain diseases, burns, poisoning, trauma or due to being in the later stages of different organ transplant surgeries such as the liver, kidney, heart, bone marrow, brain, and nerve surgeries (1). A large percentage of patients in ICUs are in a state of coma, and assessing the level of consciousness in comatose patients is an important task of these units. Body temperature and blood pressure are among available unclear criteria to assess coma. Therefore, the coma grade of patients would have been evaluated for long time based on clinical status of patients (2). Glasgow coma scale (GCS) was designed for the first time in 1974 to assess the level of consciousness in patients with traumatic brain injury (3), and then was used extensively to evaluate the level of consciousness in the other patients admitted in ICUs (4). Many studies have shown that GCS provides primary care guidelines and primary outcome prediction, morbidity, and mortality $(5,6)$. GCS disability in assessing verbal responses of intubated patients and brain stem reflexes led to the development of several scales to evaluate the level of consciousness in intubated patients (6). However, none of them have been widely used. In addition, the GCS validity in predicting outcome of disease has been put under question in many studies (7). Researchers at the Mayo Clinic in America (8) have recently designed Full Outline of Unresponsiveness (FOUR) score consisting of four components including eye responses, motor responses, brainstem reflexes, and breathing pattern. Several studies have examined the validity of the FOUR score and it has been suggested as a viable alternative to the GCS 
$(8,9)$. Idrovo et al. in a study on 60 patients with a diagnosis of stroke determined that the FOUR score is a reliable tool to assess the level of consciousness in patients with stroke; and there is a significant correlation between GCS and FOUR score in evaluating the level of consciousness of patients (10). Another study by Lyer et al. performed in ICU showed that the agreement in two GCS and FOUR score tools in the evaluation of the level of consciousness in patients was at a high level. Moreover, the mortality rate was higher among patients with low FOUR score compared to patients with low GCS score, and the FOUR score is a good predictor for determining prognosis in patients hospitalized in ICUs (8). In all the studies mentioned, taking muscle relaxant drugs, analgesics, and sedatives is one of the exclusion criteria. In addition, no study was found regarding the effect of sedation on the level of consciousness and outcome in patients.

\section{Objectives}

The present study was conducted to assess the ability of the two GCS and FOUR score tools in measuring the level of consciousness and outcome in patients receiving sedation under mechanical ventilation.

\section{Methods}

The present analytical descriptive study was conducted on adult trauma patients receiving sedation under mechanical ventilation admitted to the intensive care units in 2016 after obtaining informed consent from the first-degree relatives of patients, necessary approvals from the department of science and technology of Zahedan University of Medical Sciences in Iran, ethics committee approval, and the ethical code (IR.ZAUMS.REC.1394.201). The sample size was determined based on the previous studies and related formula as $80(11,12)$. The sample patients were selected using convenience sampling method. Inclusion criteria for the study were the age range between 12 and 65 years, connecting to mechanical ventilator, physician's order to start the infusion injection of analgesics and sedatives, no malignant and deadly disease, no diabetes, no heart, kidney, and liver failures, no damage to spinal cord in the neck area, no musculoskeletal paralysis, no deafness and blindness, as well as no history of ophthalmic surgery leading to pupil shape change.

The two tools including GCS and FOUR score were used to collect data (Figure 1). Validity and reliability were obtained on 155 patients in Tehran in 2011 through four nurses, and anesthesiologist resident, and the researcher in two trained and untrained groups, giving a very good intergroup agreement. The coefficient of agreement for the
FOUR score scale was 0.998 and 0.993 in the two trained and untrained groups, respectively. The Kappa coefficient for the intra-group agreement was 0.981 and 0.986 in the two trained and untrained groups, respectively. The coefficient of agreement between the two tools including FOUR score and GCS was 0.980 and 0.925 , respectively (13). For all patients who had inclusion criteria, a score from both GCS and FOUR score was recorded in a pre-designed form after obtaining doctor's written order for sedative infusion and before starting infusion sedation. In addition to recording the FUOR and GCS scores before starting the infusion sedation, the scores of scales were measured and recorded daily at 12 noon. These measurements were also recorded at the time of cessation of narcotic and sedative drugs use, and continued until discharge or death of the patients. Finally, the scores obtained from the study scales were compared to determine the level of ability in measuring the level of consciousness and outcome in patients in SPSS software using descriptive statistics and chi-square test for qualitative data analysis with the outcome, independent t-test to examine the association between quantitative variables in the two groups, repeated measures ANOVA to study changes in the different variables in each group, and logistic regression test to evaluate predictability of all variables with the outcome.

\section{Results}

Of 80 patients studied, 61 were male $(76.2 \%)$ and the rest were female. The mean age of the study participants was $29.2 \pm 14.7$ years. The reason for hospitalization of patients was head injuries without bleeding (35\%), head trauma with subdural hemorrhage (18.8\%), head trauma with epidural hemorrhage (16.2\%), head trauma with intracerebral hemorrhage (10\%), lower extremity trauma (7.5\%), thoracic and lumbar spinal trauma (6.2\%), and abdominal trauma (6\%), in sequence. In total, 29 (36.2\%) of the participants reported history of addiction. In terms of types of sedative drugs received, 65 patients (81.2\%) had used midazolam and fentanyl, and the rest of them had used morphine and midazolam. The mean duration of hospitalization was $14.8 \pm 8.8$ days ( 4 to 34 days), and 64 (80\%) out of 80 patients were discharged with recovery and the rest died.

Based on the results of Chi-Square test, there was no statistically significant difference in mortality between males and females $(\mathrm{P}=0.514)$; also, no statistically significant difference was found in mortality between patients with and without history of addiction $(\mathrm{P}=0.389)$.

Independent t-test results showed a statistically significant difference in the mean age between surviving (27.5 years) and deceased (36.3 years) patients $(\mathrm{P}=0.032)$, and no 


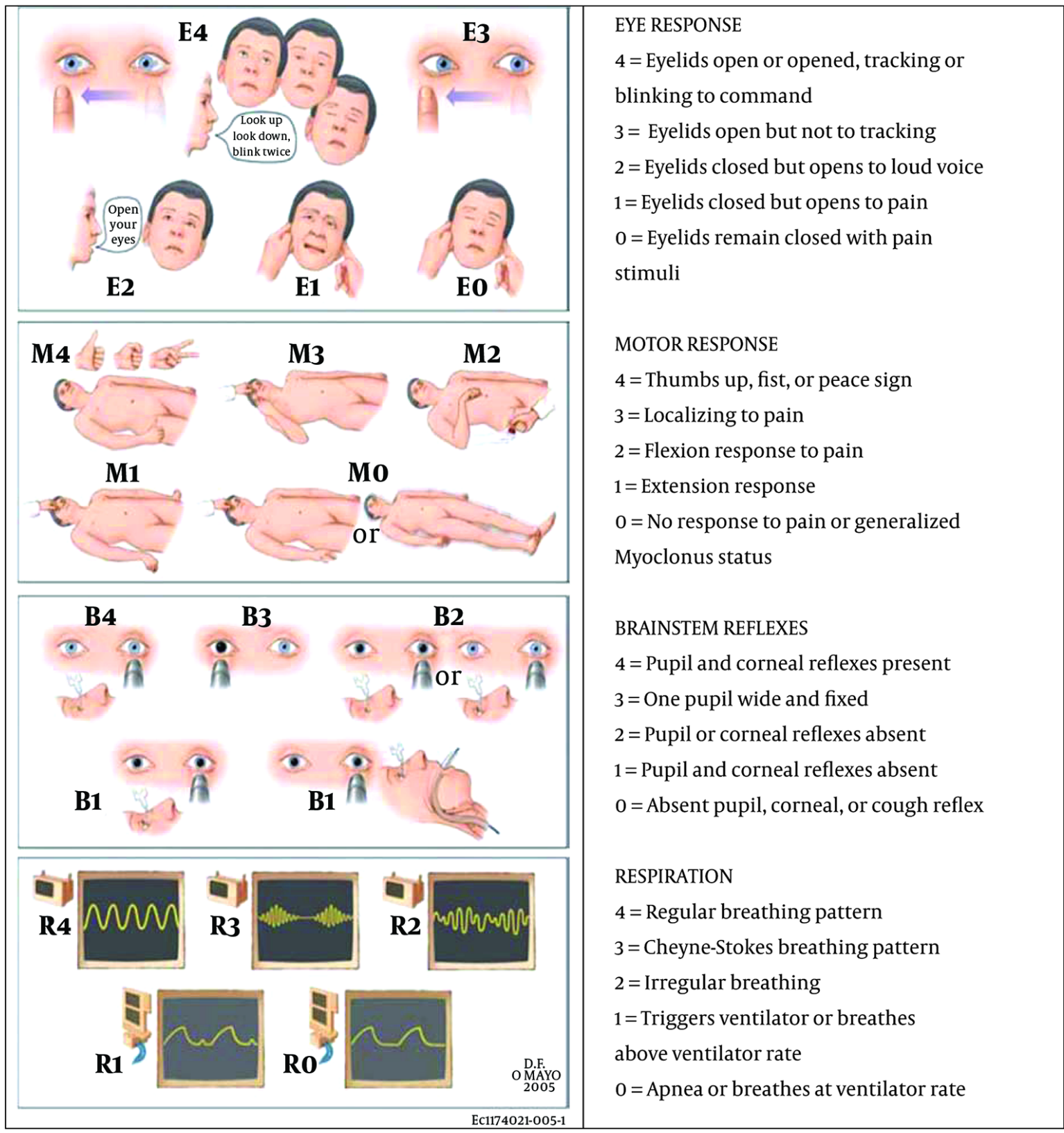

Figure 1. The two tools including GCS and FOUR score were used to collect data

statistically significant difference in the mean duration of hospitalization between surviving (15.1 days) and deceased (14.2 days) patients $(\mathrm{P}=0.588)$. The independent t-test results showed no statistically significant difference in the mean GCS score on the first day of hospitalization between females $(6 \pm 2.2)$ and males (5.9 \pm 1.2$)$, although the mean score was slightly higher in females than males $(\mathrm{P}=0.826)$.
The independent t-test results indicated a statistically significant difference in the mean GCS score on the first day of hospitalization between addicts and non-addicts, so that the mean score was significantly lower in addicts than nonaddicts (5.6 vs. 6.1) $(\mathrm{P}=0.029)$. Pearson correlation test showed a positive non-significant correlation between the mean GCS score and the mean age of patients $(\mathrm{P}=0.451$, rr 
$=0.086$ ), as well as a negative non-significant relationship between the mean GCS score and duration of hospitalization $(\mathrm{P}=0.673, \mathrm{rr}=-0.046)$.

The independent t-test results indicated no difference in the mean FOUR score on the first day of hospitalization between females (8.1) and males (8.2) although the mean score was slightly higher in males than females $(\mathrm{P}=0.925)$. The independent t-test results showed a difference in the mean FOUR score on the first day of hospitalization between addicts and non-addicts, so that the mean score was significantly lower in addicts than non-addicts (7.1 vs. 8.8) $(P=0.002)$. Pearson correlation coefficient demonstrated a negative non-significant relationship between the mean FOUR score and the mean age of patients $(\mathrm{P}=0.918, \mathrm{rr}=$ 0.012), as well as a positive non-significant correlation between the mean FOUR score and duration of hospitalization $(\mathrm{P}=0.371$, $\mathrm{rr}=0.101)$.

The mean admission GCS score was $5.9 \pm 1.2$, and the mean admission FOUR score was 8.2 \pm 3.9 . The independent t-test results revealed no statistically significant association between the mean first-day GCS score and mortality of patients although the deceased patients received higher mean score (5.8 vs. 6.3) $(\mathrm{P}=0.978)$. The independent t-test results showed no statistically significant association between the mean first-day FOUR score and mortality of patients, although the deceased patients obtained higher mean score (8.1 vs. 8.3) $(\mathrm{P}=0.578)$.

The results of repeated measures ANOVA indicated that the mean GCS score significantly increased from admission until prior to sedation onset time (5.9 to 7.3) ( $\mathrm{P}=$ 0.001). The results of repeated measures ANOVA revealed that the mean GCS score significantly decreased after the onset of sedative infusion compared to earlier (7.3 to 6.6) $(\mathrm{P}=0.001)$. The results of repeated measures ANOVA revealed that the mean GCS score after discontinuing the infusion of midazolam increased significantly compared to before (6.6 to 8.1) $(\mathrm{P}=0.001)$. The results indicated that the mean GCS score after discontinuation of narcotic analgesics increased significantly compared to before (8.1 to 9.8) $(\mathrm{P}=0.001)$. The repeated measures ANOVA showed that the mean GCS score was in ascending trend until the final stage (9.8 to 11.4) $(\mathrm{P}=0.001)$ (Figure 2 ).

According to the results of repeated measures ANOVA, the mean FOUR score significantly increased from admission until prior to sedation (8.2 to 10.4) $(\mathrm{P}=0.001)$, as well as the mean FOUR score non-significantly reduced after starting sedative infusions compared to before starting (10.4 to 10.3) $(\mathrm{P}=0.432)$. The results of repeated measures ANOVA proved that the mean FOUR score after discontinuing the infusion of benzodiazepine increased significantly compared to before (10.3 to 11.6) $(\mathrm{P}=0.001)$, as well as the mean FOUR score after discontinuation of narcotic analgesics in-

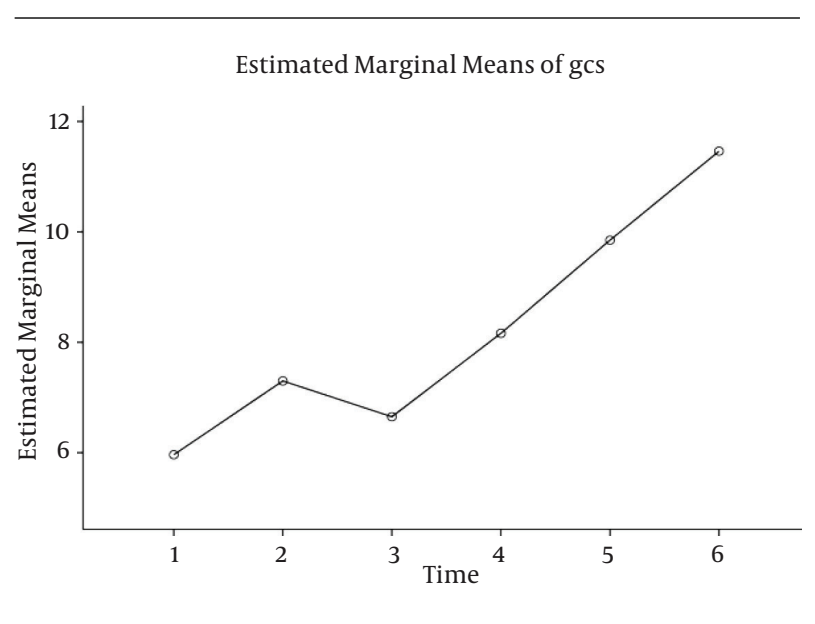

Figure 2. Estimated Marginal Means of GCS

creased significantly compared to before (11.6 to 13.2) ( $\mathrm{P}=$ 0.001). The repeated measures ANOVA indicated that the mean FOUR score was not in ascending trend until the final stage and decreased slightly (non-significantly) (13.2 to 13.1) $(\mathrm{P}=0.001)$ (Figure 3$)$.

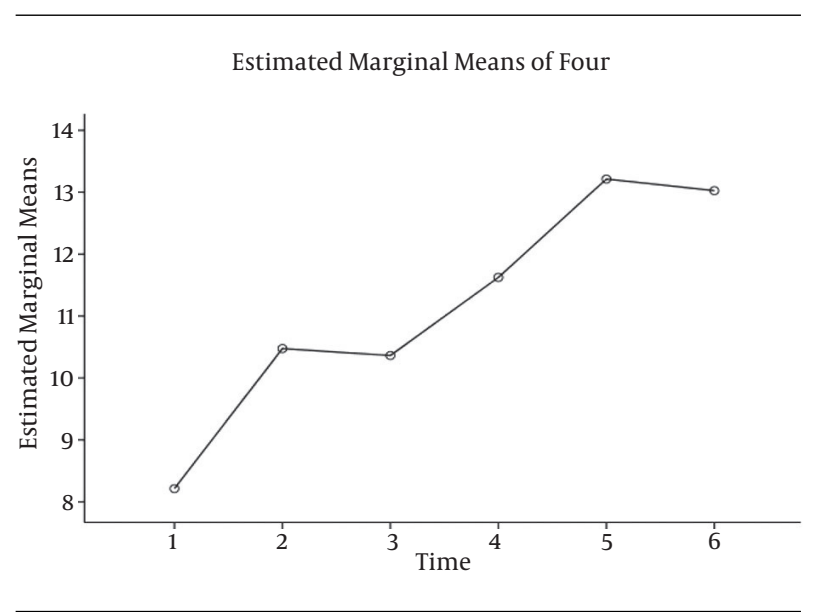

Figure 3. Estimated Marginal Means of FOUR

Based on the logistic regression analysis, only the patient age was associated with their mortality.

\section{Discussion}

The results of the present study showed that administration of sedation in trauma patients can lead to a reduction in the scores of GCS and FOUR tools, but the decline was higher in the GCS than the FOUR score. The results also indicated that the discontinuation of each of the analgesics and sedatives could cause large changes in the 
assessment scores on the level of consciousness in the patients in both scales although the changes rate was higher in the mean GCS score than the mean FOUR score. After fully discontinuing the drug, the GCS score continued to increase whereas little change was found in the mean FOUR score; according to measuring verbal response in the GCS score, this increase could be due to the cumulative effect of analgesics and sedatives and their high impact in changes on verbal response in the patients. The upward trend continued even after cessation of analgesics until 24 hours later. Jalali et al. reported that the FOUR score is better compared to GCS score to assess the level of consciousness and prediction of mortality. The results of the mentioned study in assessing the level of consciousness indicated superiority of the FOUR score that is in line with our results in the present study. Nevertheless, their findings on the prediction of mortality is in contrast to the results of the current study that shows that the low scores of scales are not related to the mortality of patients because the deceased patients had higher mean score in both scales (14). In line with the results of our study, other studies reported that the FOUR score provides better neurological details and it is not dependent on the verbal response, because the verbal response will change more under the influence of minor disorders (15). In other studies, the FOUR score has been proposed as a superior scale for measuring the level of consciousness in emergency patients (16) as well as after cardiac and respiratory arrest (17). In contrast to the results of the present study, the FOUR scale has been introduced in another study as a suitable predictor to determine the recovery and mortality rates, and it has been reported that most of the improvements occurred in patients who had the FOUR score over 10. Given that the low scores of this scale can provide better patient assessment and prediction compared to the GCS score, they recommend the use of this scale in neurosurgical patients and other patients in case of change in the level of consciousness. However, there were differences in etiology among the two study patients. Patients with tumors, aneurysms, hydrocephalus, and brain infections were also among the subjects in the mentioned study, but the present study was conducted only on patients with trauma (18). Many other factors can also affect the rate of recovery or death that were not evaluated in any of the studies, such as the skill of the surgeon, proper time of surgery, surgery and care equipment available or the incidence rate of hospital infections and their impact on mortality of patients. All the above could be the reason for the differences in results in different settings. Bakhtiari et al. reported the same ability for these two measurements in assessing the level of consciousness and mortality rate; also, they found a significant relationship between primary scores obtained from the two scales and mortality rate. Increasing the scores in the first week of hospitalization was significantly associated with the rate of improvement and reduction of neurological defects. These findings are consistent with the results of the present study, since the primary scores obtained in this study were not significantly associated with mortality rate, and the patients with higher scores on both scales died that is contrary to previous studies. However, the age range of the patients and etiology were quite different compared to the present study. Unlike the present study, they reported that none of the demographic variables had significant association with the rate of mortality and recovery; but there was a significant relationship between age and mortality rate in the current study so that older patients had higher mortality rates. The mean age of deceased patients was 36.6 years and the study patients were not elderly unlike the mentioned study (11). Other studies on the ability to predict mortality by two measurement tools suggest that no patient survives with the score of zero or one in the FOUR scale, while the patients with the mean GCS score of three improved after three months follow-up. According to brain stem reflexes assessment in the FOUR scale, the primary minimum score predicts the amount of brain death better than GCS score $(7,19)$. Other studies have also reported that the two GCS and FOUR scores have a good correlation with each other to predict mortality and recovery rates, not being in agreement with the results of our study (15).

\subsection{Conclusions}

Considering the importance of accurate measurement of consciousness level in patients and judgment of their situation based on the obtained score from the relevant scales, and with respect to the effect of analgesics injection on the scores of patients receiving sedation, scales that are less affected by the analgesics injection are suitable for measuring the level of consciousness. According to the results of this study, the FOUR score is better than the GCS o measure the level of consciousness in patients receiving sedation.

\section{Acknowledgments}

We would like to express our gratitude to the community nursing research center and the deputy of research and technology, Zahedan University of Medical Sciences, for their financial and moral support of this study.

\section{Footnotes}

Conflict of Interests: The authors declare that there is no conflict of interests regarding the publication of this pa- 
per.

Authors' Contribution: Aliakbar Keykha: data collection, drafting of the manuscript, and final revision of the manuscript. Hasan Askari: data collection and assistance in drafting of the manuscript; Ali Navidian: assistance in study design, statistical analysis; Bibi Mahdie Khodadadi Hosseini: participation in the research implementation.

\section{References}

1. Asgari M, Soleimani M. Comprehansive book intensive nursing care in ICU, CCU and dialysis. Tehran: Boshra; 2013.

2. Zand S, Rafiei M. Causes and duration of hospitalization and mortality rate in intensive care units in Central province. J Crit Care Nurs. 2010;3(2):7-8.

3. Teasdale G, Jennett B. Assessment of coma and impaired consciousness. A practical scale. Lancet. 1974;2(7872):81-4. [PubMed: 4136544].

4. Gill M, Martens K, Lynch EL, Salih A, Green SM. Interrater reliability of 3 simplified neurologic scales applied to adults presenting to the emergency department with altered levels of consciousness. Ann Emerg Med. 2007;49(4):403-7. doi: 10.1016/j.annemergmed.2006.03.031. [PubMed: 17141146] 407 e1.

5. Gill MR, Reiley DG, Green SM. Interrater reliability of Glasgow Coma Scale scores in the emergency department. Ann Emerg Med. 2004;43(2):215-23. doi: 10.1016/S019606440300814X. [PubMed: 14747811].

6. Khajeh A, Fayyazi A, Miri-Aliabad G, Askari H, Noori N, Khajeh B. Comparison between the Ability of Glasgow Coma Scale and Full Outline of Unresponsiveness Score to Predict the Mortality and Discharge Rate of Pediatric Intensive Care Unit Patients. Iran J Pediatr. 2014;24(5):603-8. [PubMed: 25793069].

7. Fischer M, Ruegg S, Czaplinski A, Strohmeier M, Lehmann A, Tschan $\mathrm{F}$, et al. Inter-rater reliability of the Full Outline of UnResponsiveness score and the Glasgow Coma Scale in critically ill patients: a prospective observational study. Crit Care. 2010;14(2):R64. doi: 10.1186/cc8963. [PubMed: 20398274].

8. Iyer VN, Mandrekar JN, Danielson RD, Zubkov AY, Elmer JL, Wijdicks EFM, editors. Validity of the FOUR score coma scale in the medical intensive care unit. Mayo Clinic Proceedings. 2009; Elsevier; pp. 694701
9. Akavipat P, Sookplung P, Kaewsingha P, Maunsaiyat P. Prediction of discharge outcome with the full outline of unresponsiveness (FOUR) score in neurosurgical patients. Acta Med Okayama. 2011;65(3):205-10. [PubMed: 21709719].

10. Idrovo L, Fuentes B, Medina J, Gabaldon L, Ruiz-Ares G, Abenza MJ, et al. Validation of the FOUR Score (Spanish Version) in acute stroke: an interobserver variability study. Eur Neurol. 2010;63(6):364-9. doi: 10.1159/000292498. [PubMed: 20551672].

11. Bakhtiari S, Amiri F, Kazemnegad A, Bakhshandeh H, Mohamadaliha Z, Termechi G, et al. Comparison gcs and four score for predicting neurological outcomes in patients with impairment of consciousness. ; 2014.

12. Buyukcam F, Kaya U, Karakilic ME, Cavus UY, Turan Sonmez F, Odabas O. Predicting the outcome in children with head trauma: comparison of FOUR score and Glasgow Coma Scale. Ulus Travma Acil Cerrahi Derg. 2012;18(6):469-73. doi:10.5505/tjtes.2012.23169. [PubMed: 23588903].

13. Tadrisi SD, Bahari N, Ebadi A, Madani SJ. Validity and reliability of coma scale (four score) in adult patient hospitalized in Critical Care Units. Journal of Critical Care Nursing. 2012;5(2):95-102.

14. Jalali R, Rezaei M. A comparison of the Glasgow Coma Scale score with full outline of unresponsiveness scale to predict patients' traumatic brain injury outcomes in intensive care units. Critical Care Res Pract. 2014;2014.

15. Bruno MA, Ledoux D, Lambermont B, Damas F, Schnakers C, Vanhaudenhuyse A, et al. Comparison of the Full Outline of UnResponsiveness and Glasgow Liege Scale/Glasgow Coma Scale in an intensive care unit population. Neurocrit Care. 2011;15(3):447-53. doi:10.1007/s12028011-9547-2. [PubMed: 21526394].

16. Ledoux D, Bruno M, Jonlet S, Choi P, Schnakers C, Damas F, et al. Full Outline of Unresponsiveness compared with Glasgow coma scale assessment and outcome prediction in coma. Crit Care. 2009;13(Suppl 1):P107. doi: 10.1186/cc7271.

17. Kevric J, Jelinek GA, Knott J, Weiland TJ. Validation of the Full Outline of Unresponsiveness (FOUR) Scale for conscious state in the emergency department: comparison against the Glasgow Coma Scale. Emerg Med J. 2010:emj. 2009.085845.

18. Phuping A, Pimwan S, Pranee K, Patcharin M. Prediction of Discharge Outcome with the Full Outline of Unresponsiveness (FOUR) Score in Neurosurgical Patients. ; 2011.

19. Fugate JE, Rabinstein AA, Claassen DO, White RD, Wijdicks EF. The FOUR score predicts outcome in patients after cardiac arrest. Neurocrit Care. 2010;13(2):205-10. doi: 10.1007/s12028-010-9407-5. [PubMed: 20645026]. 